\title{
A re-examination of the impact of object processing on shifts of spatial attention
}

\author{
Troy A. W. Visser
}

Published online: 17 November 2010

(C) Psychonomic Society, Inc. 2010

\begin{abstract}
When two targets are presented in rapid succession at the same spatial location, processing of the first is highly efficient, while processing of the second is often profoundly impaired at brief inter-target intervals (attentional blink; $\mathrm{AB}$ ). While the $\mathrm{AB}$ has been shown to impact many processes, it is still unclear whether this includes the ability to shift spatial attention. The present study examined this question using a more sensitive dependent measure than past studies; namely, response times. It also evaluated whether masking of the cue stimulus modulated the effect of the $\mathrm{AB}$ on spatial shifts. The results showed significant cueing effects on $\mathrm{T} 2$ response times that were strongly modulated by the AB. This supports suggested links between mechanisms underlying object processing and spatial shifts of attention.
\end{abstract}

Keywords Attentional blink - Spatial cueing - Attention . Orienting

\section{Introduction}

Although our brains are fabulously complex computational devices, decades of research suggest they are also subject to profound limitations in their information processing capabilities. One such limitation can be seen when observers attempt to identify two consecutive targets: while firsttarget (T1) identification is nearly perfect, second-target (T2) identification is typically impaired at inter-target intervals (lags) of less than about $500 \mathrm{~ms}$. This T2 deficit is known as the attentional blink (AB).

T. A. W. Visser $(\bowtie)$

School of Psychology, University of Queensland,

St. Lucia, QLD 4072, Australia

e-mail: taw.visser@gmail.com
Theories universally attribute the $\mathrm{AB}$ to $\mathrm{T} 1$ processing. This is variously thought to limit memory encoding of T2 (Chun \& Potter, 1995; Jolicoeur, 1998), deplete cognitive resources (Duncan, Ward, \& Shapiro, 1994), lead to suboptimal input filtering (Di Lollo, Kawahara, Ghorashi, \& Enns, 2005), trigger inhibition (Raymond, Shapiro, \& Arnell, 1992; Olivers \& Meeter, 2008), or impair transient attention (Bowman \& Wyble, 2007). What is not clear, however, is whether object processing at one spatial location impairs shifts of spatial attention to a different location. This issue speaks directly to the relationship between mechanisms underlying temporal and spatial attention.

One approach to addressing this issue has been to present $\mathrm{T} 1$ and $\mathrm{T} 2$ at different spatial locations, and then examine whether this leads to behavioral or electrophysiological changes consistent with an $\mathrm{AB}$. Using this approach, Joseph, Chun, and Nakayama (1997) showed reduced search efficiency for a pop-out peripheral target at shorter lags. Similarly, Visser, Zuvic, Bischof, and Di Lollo (1999) obtained a substantial AB when observers identified two masked targets presented at different locations (see also Duncan et al., 1994; Kristjansson \& Nakayama, 2002). Finally, Jolicoeur and colleagues (Dell'Acqua, Sessa, Jolicoeur, \& Robitaille, 2006; Jolicoeur, Sessa, Dell'Acqua, \& Robitaille, 2006; Robitaille, Jolicoeur, Dell'Acqua, \& Sessa, 2007) indexed spatial shifts of attention during the $\mathrm{AB}$ using the $\mathrm{N} 2 \mathrm{pc}$ component obtained while recording event-related potentials (ERPs) to peripheral targets presented at varying lags after a central T1. They found decreased N2pc magnitude during the $\mathrm{AB}$, suggesting that spatial attention was "frozen" during object processing (Dell'Acqua et al., 2006).

Another approach to this question has been to examine how object processing impacts shifts of spatial attention to 
a visual cue presented prior to $\mathrm{T} 2$ onset. ${ }^{1}$ This methodology has yielded mixed results. For example, Ghorashi and colleagues (Ghorashi, Di Lollo, \& Klein, 2007; Ghorashi, Enns, Klein, \& Di Lollo, 2010; Ghorashi, Enns, Spalek, \& Di Lollo, 2009; Ghorashi, Spalek, Enns, \& Di Lollo, 2009) repeatedly failed to find an interaction between the $\mathrm{AB}$ and exogenous, endogenous, predictive and non-predictive visual cues. In contrast, Du and Abrams (2009) presented observers with a central target, followed by a nonpredictive cue, and a single peripheral target, and found progressively greater cueing effects as T1-cue interval increased (for similar results, see Nieuwenstein, Chun, van der Lubbe, \& Hooge, 2005; Olivers, 2004).

Examination of the studies above suggests at least two possible reasons for their discrepant outcomes. The first is backward masking which refers to a decrease in the visibility of a target caused by presentation of a second temporally trailing stimulus called a mask (Breitmeyer \& Ogmen, 2006). In different-locations studies, the impact of the $\mathrm{AB}$ was assessed on spatial shifts to a backwardmasked T2 (omission of this mask usually eliminates the AB; Giesbrecht \& Di Lollo, 1998). On the other hand, in cuing studies, the impact of the $\mathrm{AB}$ was assessed on spatial shifts to a cue, which was rarely masked. ${ }^{2}$ The potential impact of this difference is highlighted by the work of Olivers (2004), who obtained a reliable effect of the AB on cuing when the cue display was masked (Experiments 1 and 2), but not when the mask was omitted (Experiment 5).

A second possibility is that previous studies did not use a sufficiently sensitive dependent measure to detect the impact of the $\mathrm{AB}$ on cuing. Other than Olivers (2004), all previous studies have assessed the impact of the cue on T2 identification using accuracy or critical T2-mask interval as the dependent measure. While this is typical in $\mathrm{AB}$ studies, it contrasts with the majority of spatial cuing and visual search studies that have used response times (RTs) to assess cueing effects (e.g., Folk, Remington, \& Johnston, 1992; Jonides, 1981; Posner, 1980). To determine the relevance of

\footnotetext{
${ }^{1}$ Pashler (1991) employed a similar approach in an experiment requiring immediate response to $\mathrm{T} 1$. Here, no relationship was found between $\mathrm{T} 1$ processing and cueing. It seems likely that the differences between these results and the $\mathrm{AB}$ studies outlined here are due to the fact that the speeded $\mathrm{T} 1$ response tapped motor selection processes more than an unspeeded task. Differences between Pashler's results and those reported here may thus speak to the role of motor selection in central resource limitations underlying the $\mathrm{AB}$ (e.g., Dell'Acqua \& Jolicoeur, 2000; Jolicoeur, 1998, 1999), and to the nature of the overlap between mechanisms underlying the $\mathrm{AB}$ and the psychological refractory period (e.g., Marois \& Ivanoff, 2007).

${ }^{2}$ It is possible that some masking could have arisen from the target on cued trials. However, in many experiments, mask-target contours were dissimilar (e.g., Ghorashi et al., 2010), thus limiting masking strength. In addition, cueing effects have also been obtained using endogenous cues (Ghorashi, Spalek, et al., 2009) where the cue was not presented at the target location.
}

this difference, T2 RTs were measured to assess the impact of the $\mathrm{AB}$ on spatial cuing.

\section{Experiment 1}

Broadly replicating the work of Ghorashi and colleagues, observers viewed a central T1 letter target, which was followed after a variable interval by a peripheral cue that was either masked or not. The cue was non-predictive, and was followed by the appearance of $\mathrm{T} 2$ (' $\mathrm{C}$ ' or ' $\mathrm{G}$ '). Observers made a speeded two-alternative decision about the identity of $\mathrm{T} 2$, followed by a non-speeded identification of T1.

Participants Eighteen undergraduate psychology students (12 female) completed the experiment for course credit. All reported normal or corrected-to-normal vision.

Apparatus Stimuli were presented on 19-inch (c.48.3-cm) ViewSonic CRT monitors (Model G90ft+) running at a refresh rate of $100 \mathrm{~Hz}$ controlled by Pentium-4 computers running Presentation software (Neurobehavioral Systems). Participants were seated approximately $60 \mathrm{~cm}$ from the monitor in a quiet, dark room, with only dim keyboard illumination from a small light.

Method The experiment consisted of two conditions, presented in counterbalanced order, each comprising 220 trials, divided into 11 blocks of 20 trials. The first block of trials in each condition was treated as practice and was not analyzed. Trials consisted of all possible factorial combinations of T1$\mathrm{T} 2$ lag and T2 peripheral location. As part of an experimental manipulation unrelated to the present work, the number of lag-1 trials, in which $\mathrm{T} 1$ and $\mathrm{T} 2$ appeared directly after one another, comprised $60 \%$ of trials, while lags 3 and 7 comprised $20 \%$ of trials respectively.

The sequence of events on a typical trial is depicted in Fig. 1. Each trial began with a fixation cross $\left(0.3^{\circ} \times 0.3^{\circ}\right.$; RGB: $128,128,128)$ at the centre of the display, flanked by placeholder boxes $\left(2^{\circ} \times 2^{\circ}\right.$; RGB: $\left.136,136,136\right)$ to the right and left (centre-to-centre separation: $10^{\circ}$ ). Participants were instructed to maintain eye gaze at fixation throughout the trial, and to press the spacebar to begin. This initiated a central stream consisting of 5 - digits $\left(1^{\circ} \times 1^{\circ}\right.$; RGB: 250 , $250,250)$ displayed for $10 \mathrm{~ms}$ each and followed by a 70 ms inter-stimulus interval (ISI) during which fixation and placeholders were displayed. These digits were followed by the presentation of T1 (randomly-chosen letter; $1^{\circ} \times 1^{\circ}$; RGB: 250, 250, 250; I, O, Q, Z, C, G omitted) for $10 \mathrm{~ms}$.

The cue stimulus (' $\mathrm{X}$ '; $1.5^{\circ} \times 1.5^{\circ}$; RGB: $210,210,210$ ) appeared either directly after $\mathrm{T} 1$, or was separated from $\mathrm{T} 1$ by an additional two or six digit distractors (temporal 
Fig. 1 Schematic illustration of the sequence of events on a Lag 3 trial in Experiment 1 (not to scale). Participants identified a single letter presented amongst a rapid-serial visual stream of digits, and then decided whether a second peripheral target was the letter ' $G$ ' or ' $C$ '. The peripheral letter was preceded by a non-predictive cue (' $\mathrm{X}$ ') presented in one of the two possible target locations. In one block of trials, a mask followed the cue in each possible location; this mask was omitted in the other block

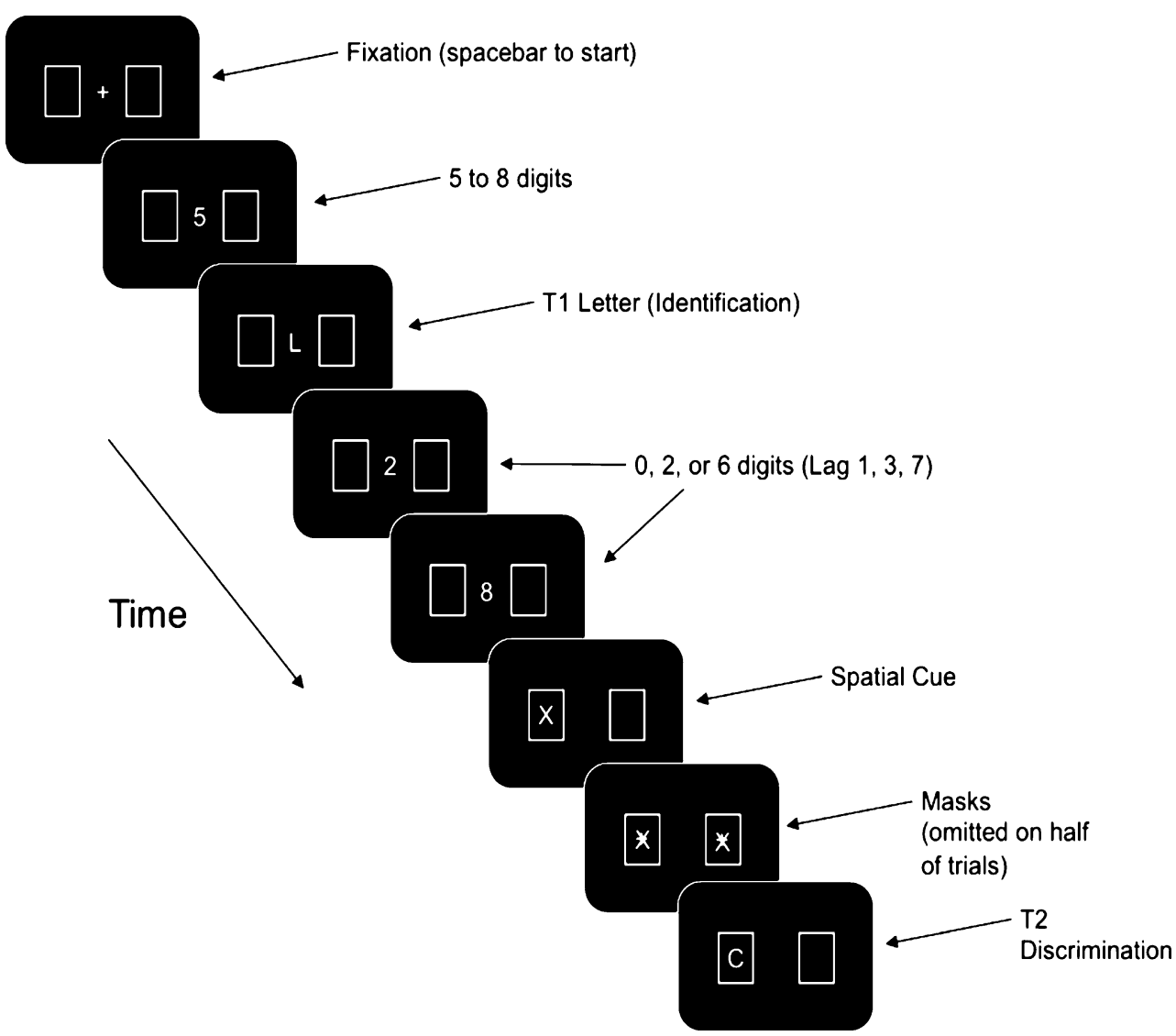

intervals: 80, 240, $560 \mathrm{~ms}$; lags 1, 3, 7), and was presented inside a placeholder for $10 \mathrm{~ms}$. Participants were instructed to ignore the cue because it did not predict T2 location. The cue was followed by a 70-ms ISI, and a mask stimulus consisting of a superimposed outline square, ' $\mathrm{X}$ ', and ' + ' sign $\left(1.5^{\circ} \times 1.5^{\circ}\right.$; RGB: $\left.250,250,250\right)$ for $10 \mathrm{~ms}$ (masked condition), or an $80 \mathrm{~ms}$ blank display (non-masked condition). Finally, T2 ('C' or ' $G$ '; $1^{\circ} \times 1^{\circ}$; RGB: 250, 250, 250) was presented inside a placeholder for $2,000 \mathrm{~ms}$ or until response. Participants were instructed to identify T2 as quickly as possible by pressing an appropriately marked key, and then were prompted to type the T1 letter at their leisure. After responding, the fixation cross re-appeared, and participants began the next trial by pressing the spacebar.

On $20 \%$ of trials, the target was omitted, with the fixation and peripheral boxes alone displayed for 2,000 ms, followed by the prompt to report the identity of $\mathrm{T} 1$. These "catch trials" ensured that observers did not try to anticipate target onset.

\section{Results}

T1 identification accuracy Mean accuracy scores were submitted to a T1-T2 Lag $(1,3,7) \times$ Cue Mask (Present,
Absent) within-subjects ANOVA. No main effects or interactions were significant (all $p \mathrm{~s}>.20$, all $\eta^{2} \mathrm{~s}<.09$ ). Overall T1 accuracy was $90.60 \%$.

Catch trial accuracy Mean catch trial accuracy was $94.20 \%$ on trials in which the cue was masked, and $93.22 \%$ on trials on which the cued was not masked, $t(17)=1.40, p>.17$.

T2 identification accuracy Mean accuracy was on T1correct trials (see Table 1; Fig. 2) was submitted to a Lag $\times$ Cue Mask x Cue Validity within-subjects ANOVA. Only a Lag $\times$ Cue Mask interaction was obtained, $F(2,34)=3.51$, $p<.05, \eta^{2}=.18$, indicating that accuracy trended higher

Table 1 Mean T2 Accuracy in Experiment 1 as a function of Cue Validity, Presence/Absence of the Cue mask, and T1-T2 Lag. Numbers in brackets represent one standard error of the mean

\begin{tabular}{lllll}
\hline Cue mask & Cue Validity & \multicolumn{3}{l}{ Lag } \\
\cline { 3 - 5 } & & 1 & 3 & 7 \\
\hline \multirow{2}{*}{ Present } & Valid & $84.2(4.4)$ & $88.6(4.6)$ & $89.0(4.6)$ \\
& Invalid & $87.7(3.7)$ & $86.8(5.4)$ & $90.6(4.1)$ \\
Absent & Valid & $86.1(4.4)$ & $85.6(5.1)$ & $85.0(5.6)$ \\
& Invalid & $85.1(4.3)$ & $86.7(4.7)$ & $84.3(4.2)$ \\
\hline
\end{tabular}




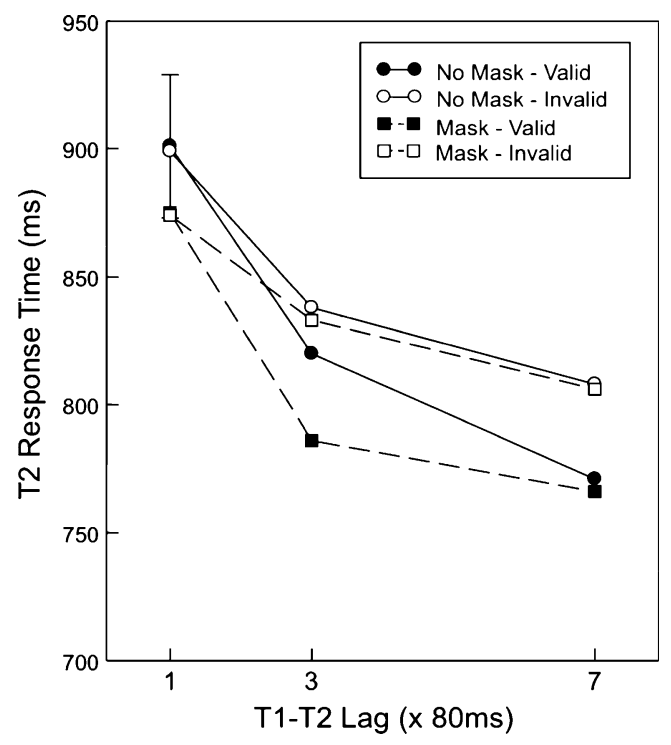

Fig. 2 T2 response times as a function of inter-target lag, cue masking and cue validity on trials in which $\mathrm{T} 1$ was accurately identified in Experiment 1. Error bars 95\% within-subjects confidence intervals calculated as per Masson and Loftus (2003)

across lags when the cue was masked, but lower when the mask was absent. Notably, as in the work of Ghorashi and colleagues, there was no interaction between Lag and Cue Validity $\left(p>.75, \eta^{2}<.02\right)$.

T2 RTs Mean RTs on T2-correct trials were submitted to a Lag $\times$ Cue Mask $\times$ Cue Validity within-subjects ANOVA which revealed main effects of Lag, $F(2,34)=48.86, p<$ $.001, \eta^{2}=.74$, and Cue Validity, $F(1,17)=12.38, p<.01$, $\eta^{2}=.42$. This reflects a decline in RTs with increasing Lag, and a valid cuing benefit of approximately $23 \mathrm{~ms}$. Importantly, there was also a significant Lag $\times$ Cue Validity interaction, $F(2,34)=3.69, p<.04, \eta^{2}=.18$., reflecting increased cue validity effects as lag increased.. Also notable, was the absence of any interactions between masking and Cue Validity $\left(p \mathrm{~s}>.55, \eta^{2} \mathrm{~s}<.03\right)$.

The present results invite two conclusions. First, the presence or absence of a cue mask does not modulate the impact of the $A B$ on cueing. Such effects were obtained whether the cue was masked or not. Second, it seems that the choice of dependent measure used to assess cueing effects is important. Whereas this and many previous experiments have failed to find an interaction between cueing and the $\mathrm{AB}$ using identification accuracy, just such an interaction was found using RTs.

The purpose of Experiment 2 was to address a possible alternative explanation for the results. Ghorashi, Spalek, et al. (2009) argued that spatial cueing should interact with the $\mathrm{AB}$ if both the cue and the target required common processing mechanisms. Indeed, they obtained this interaction when both the cue and target required observers to make a discrimination judgment. In Experiment 1, although told to ignore it, it is possible that observers obligatorily identified the familiar ' $\mathrm{X}$ ' cue, thus leading to similar processing requirements for the cue and $\mathrm{T} 2$. To eliminate this possibility, in Experiment 2, we replicated the present methodology but changed the T2 task to detection of a bright dot (see Ghorashi, Enns, et al., 2009 for a similar combination of cue-T2 events).

\section{Experiment 2}

Participants Twenty-nine undergraduate psychology students (20 female) from the same participant pool as Experiment 1 completed the experiment.

Apparatus Apparatus were identical to those used in Experiment 1.

Method The method was identical to Experiment 1, except that the T2 letter was replaced with a small, white filled square target, which observers responded to as quickly as possible by pressing the space bar.

\section{Results}

T1 identification accuracyMean accuracy was submitted to a Lag $\times$ Cue Mask within-subjects ANOVA. No main effects or interactions were significant (all $p \mathrm{~s}>.08$, all $\left.\eta^{2} \mathrm{~s}<.09\right)$. Overall $\mathrm{T} 1$ accuracy was $94.35 \%$.

Catch trial accuracy Mean catch trial accuracy was $86.02 \%$ on trials in which the cue was masked, and $87.21 \%$ on trials on which the cue was not masked, $t(28)=0.44, p>.66$.

T2 response times Only trials on which $\mathrm{T} 1$ was correctly identified were used in this analysis. In addition, trials on which RTs were less than $200 \mathrm{~ms}$ or greater than $1,000 \mathrm{~ms}$ were deemed as outliers and omitted from the analysis. Overall, this resulted in the exclusion of $2.60 \%$ of trials, with no differences as a function of Lag, Cue Mask or Cue Validity (all $p \mathrm{~s}>.05, \eta^{2} \mathrm{~s}<.10$ ).

Mean RTs on the remaining trials were submitted to a Lag $\times$ Cue Mask $\times$ Cue Validity within-subjects ANOVA which revealed main effects of Lag, $F(2,56)=83.61, p<$ $.001, \eta^{2}=.75$, and Cue Validity, $F(1,25)=5.42, p=.027$, $\eta^{2}=.16$, reflecting declining RTs with increasing lag, and a positive benefit for cued targets of approximately $8 \mathrm{~ms}$. Most importantly, the interaction between Lag and Cue Validity was significant, $F(2,56)=3.24, p=.047$, $\eta^{2}=.10$. 
As can be seen in Fig. 3, this significant interaction reflected the fact that a strong cueing benefit emerged as lag increased. Replicating the results of Experiment 1, this suggests that the $\mathrm{AB}$ modulated spatial shifts of attention to the cue. Also replicating Experiment 1, there was no indication that cue masking modulated cuing effects ( $p s>$ $\left..24, \eta^{2} \mathrm{~s}<.05\right)$.

\section{General discussion}

The present experiment addressed an issue of significant recent interest: does high-level object processing impact spatial shifts of attention? A review of past studies suggests evidence both for and against such a relationship, with studies employing a spatial cue in particular, generally failing to find an effect of object processing on spatial cueing. Here, we examined whether this lack of uniformity might stem from two particular aspects of the experimental design of prior cueing studies: (1) the use of a backward mask following the cue; and (2) the use of T2 accuracy to assess cueing effects, rather than a more sensitive RT measure.

The results were quite clear. First, robust cueing effects were seen in the RT data across both $\mathrm{T} 2$ discrimination and detection tasks. More critically, the benefit of valid cues increased with T1-cue interval. This indicates that object processing interferes with shifts of spatial attention. Second, the relationship between $\mathrm{T} 1$ processing and cueing seen in the RT data was unaffected by the presence of a

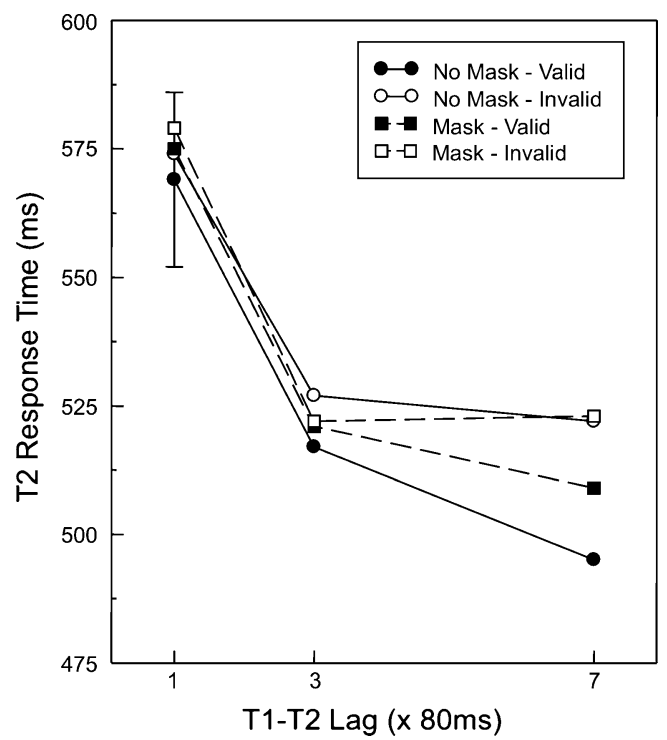

Fig. 3 T2 response times as a function of inter-target lag, cue masking and cue validity on trials in which $\mathrm{T} 1$ was accurately identified in Experiment 2. Error bars 95\% within-subjects confidence intervals calculated as per Masson and Loftus (2003) mask following the cue. Instead, the evidence suggests that previous failures to find $\mathrm{AB}$-related modulations of cueing effects arose from the use of accuracy to assess these effects rather than RTs (e.g., Posner, 1980).

In previous papers, Ghorashi and colleagues have posited that the $\mathrm{AB}$ does not impact spatial attention because object identification involves processing along the ventral visual pathway, while cue processing occurs along the dorsal pathway. Thus, unless the cue and $\mathrm{T} 2$ require common ventral or dorsal processing, $\mathrm{T} 1$ processing should not impact cueing. In contrast, the present evidence indicates that object processing "freezes" spatial attention (Dell'Acqua et al., 2006; Du \& Abrams, 2009; Olivers, 2004), thus implying considerable overlap in the mechanisms underlying object and spatial vision.

This suggestion is consistent with a number of lines of neurophysiological evidence. First, there is substantial overlap between areas that represent object properties such as shape and size and dorsal areas involved in spatial vision and attention (e.g., Konen \& Kastner, 2008). Second, the $\mathrm{AB}$ is prolonged in visual neglect patients (Husain, Shapiro, Martin, \& Kennard, 1997; Shapiro, Hillstrom, \& Husain, 2002; Van Vleet \& Robertson, 2006), implicating common pathways for the two deficits. Finally, studies have linked the $\mathrm{AB}$ with neural activity in the intraparietal sulcus (IPS; Marois, Chun, \& Gore, 2000; Kihara et al., 2007), and the temporo-parietal junction (TPJ; Gross et al., 2004; Marois, Yi, \& Chun, 2004). In turn, IPS has been strongly implicated in endogenous (goal-directed) spatial shifts of attention (e.g., Behrmann, Geng, \& Shomstein, 2004; Corbetta \& Shulman, 2002; Coull \& Nobre, 1998), while TPJ has been linked with exogenous (stimulus-driven) spatial shifts (Corbetta, Patel, \& Shulman, 2008; Geng \& Mangun, 2008). Considered together, this body of work strongly points to common neural substrates for object processing and spatial attention shifts.

Before concluding, it is worthwhile to discuss two other aspects of the present results. First, it is notable that T2 accuracy is robustly modulated by $\mathrm{T} 1$ processing, implying an effect of object processing on spatial attention, yet similar effects on T2 accuracy are not seen in spatial cueing paradigms like those used here. A likely explanation for this difference is that the $\mathrm{T} 2$ deficit in different-location studies actually reflects two types of interference from $\mathrm{T} 1$ : one on spatial shifts of attention, and the other on $\mathrm{T} 2$ identification. While the combination of these effects is detectable using accuracy measures, interference with spatial shifts alone is proportionally subtler and thus requires a more sensitive measure.

In addition, it may seem surprising that the $\mathrm{AB}$ influences exogenous cueing, a stimulus-driven effect that has classically been considered as "automatic" (e.g., Jonides, 1981; Posner, 1980). However, as noted above, 
there is evidence for a neuroanatomical overlap between areas involved in object processing during the $A B$, and exogenous orienting. Moreover, a number of studies have shown conditions under which attentional capture by exogenous cues is attenuated (e.g., Santangelo, Olivetti Belardinelli, \& Spence, 2007; Theeuwes, 1991; Yantis \& Jonides, 1990; see Santangelo \& Spence, 2008 for a review). Together, this evidence suggests that processing of exogenous cues may be impacted by intention and concurrent task performance, consistent with the effects of object processing obtained in the current experiments.

In conclusion, it will be important for future work to more closely characterize the behavioral and neurophysiological overlap between object processing and spatial attention. In particular, it will be crucial to determine which aspects of object processing and spatial orienting processes interfere, and which do not. An initial approach to this issue may be to determine whether object processing also affects endogenous cueing. This has been a matter of some uncertainty (e.g., Ghorashi, Spalek, et al., 2009; Zhang, Shao, Nieuwenstein, \& Zhou, 2008), and its resolution may help to shed light on the issues raised here.

Acknowledgments The author was supported by a UQ Early Career Research Grant. The author would like to thank Thomas Spalek, Roberto Dell'Acqua and an anonymous reviewer for helpful comments on previous versions of this manuscript.

\section{References}

Behrmann, M., Geng, J. J., \& Shomstein, S. (2004). Parietal cortex and attention. Current Opinion in Neurobiology, 14, 212-217.

Bowman, H., \& Wyble, B. P. (2007). The simultaneous type, serial token model of temporal attention and working memory. Psychological Review, 114, 38-70.

Breitmeyer, B., \& Ogmen, H. (2006). Visual masking: Time slices through conscious and unconscious vision. Oxford University Press: London.

Chun, M. M., \& Potter, M. C. (1995). A two-stage model for multiple target detection in rapid serial visual presentation. Journal of Experimental Psychology: Human Perception and Performance, 21, 109-127.

Corbetta, M., Patel, G., \& Shulman, G. L. (2008). The reorienting system of the human brain: From environment to theory of mind. Neuron, 58, 306-324.

Corbetta, M., \& Shulman, G. L. (2002). Control of goal-directed and stimulus-driven attention in the brain. Nature Reviews. Neuroscience, 3, 201-215.

Coull, J. T., \& Nobre, A. C. (1998). Where and when to pay attention: The neural systems for directing attention to spatial locations and to time intervals as revealed by both PET and fMRI. The Journal of Neuroscience, 18, 7426-7435.

Dell'Acqua, R., Sessa, P., Jolicoeur, P., \& Robitaille, N. (2006). Spatial attention freezes during the attentional blink. Psychophysiology, 43, 394-400.

Dell'Acqua, R., \& Jolicoeur, P. (2000). Visual encoding of patterns is subject to dual-task interference. Memory \& Cognition, 28, 184-191.
Di Lollo, V., Kawahara, J., Ghorashi, S., \& Enns, J. (2005). The attentional blink: Resource depletion or temporary loss of control? Psychological Research, 69, 191-200.

Du, F., \& Abrams, R. A. (2009). Onset capture requires attention. Psychonomic Bulletin \& Review, 16, 537-541.

Duncan, J., Ward, R., \& Shapiro, K. (1994). Direct measurement of attentional dwell time in human vision. Nature, 369, 313-315.

Folk, C. L., Remington, R. W., \& Johnston, J. C. (1992). Involuntary covert orienting is contingent on attentional control settings. Journal of Experimental Psychology: Human Perception and Performance, 18, 1030-1044.

Geng, J. J., \& Mangun, G. R. (2008). Anterior intraparietal sulcus is sensitive to bottom-up attention driven by stimulus salience. Journal of Cognitive Neuroscience, 21, 1584-1601.

Ghorashi, S. M. S., Di Lollo, V., \& Klein, R. M. (2007). Attentional orienting in response to peripheral cues survives the attentional blink. Visual Cognition, 15, 87-90.

Ghorashi, S. M. S., Enns, J. T., Klein, R. M., \& Di Lollo, V. (2010). Spatial selection and target identification are separable processes in visual search. Journal of Vision, 10, 1-12.

Ghorashi, S. M. S., Enns, J. T., Spalek, T. M., \& Di Lollo, V. (2009). Spatial cueing does not affect the magnitude of the attentional blink. Attention, Perception, \& Psychophysics, 71, 989-993.

Ghorashi, S. M. S., Spalek, T. M., Enns, J. T., \& Di Lollo, V. (2009). Are spatial selection and identity extraction separable when attention is controlled exogenously? Attention, Perception, \& Psychophysics, 71, 1233-1240.

Giesbrecht, B., \& Di Lollo, V. (1998). Beyond the attentional blink: Visual masking by object substitution. Journal of Experimental Psychology: Human Perception and Performance, 24, 1454 1466.

Gross, J., Schmitz, F., Schnitzler, A., Kessler, K., Shapiro, K. L., Hommel, B., et al. (2004). Modulation of long-range neural synchrony reflects temporal limitations of visual attention in humans. Proceedings of the National Academy of Science, 101, 13050-13055.

Husain, M., Shapiro, K. L., Martin, J., \& Kennard, C. (1997). Abnormal temporal dynamics of visual attention in spatial neglect patients. Nature, 385, 154-156.

Jolicoeur, P. (1998). Modulation of the attentional blink by online response selection: Evidence from speeded and unspeeded task1 decisions. Memory \& Cognition, 26, 1014-1032.

Jolicoeur, P. (1999). Concurrent response-selection demands modulate the attentional blink. Journal of Experimental Psychology: Human Perception and Performance, 25, 1097-1113.

Jolicoeur, P., Sessa, P., Dell'Acqua, R., \& Robitaille, N. (2006). On the control of visual spatial attention: Evidence from electrophysiology. Psychological Research, 70, 414-424.

Jonides, J. (1981). Voluntary versus automatic control over the mind's eye's movement. In J. B. Long \& A. D. Baddeley (Eds.), Attention \& Performance (Vol. 9). Hillsdale, N.J.: Erlbaum.

Joseph, J. S., Chun, M. M., \& Nakayama, K. (June 19, 1997). Attentional requirements in a 'preattentive' feature search task. Nature, 387, 805-807.

Kihara, K., Hirose, N., Mima, T., Abe, M., Fukuyama, H., \& Osaka, N. (2007). The role of left and right intraparietal sulcus in the attentional blink: A transcranial magnetic stimulation study. Experimental Brain Research, 178, 135-140.

Konen, C. S., \& Kastner, S. (2008). Two hierarchically organized neural systems for object information in human visual cortex. Nature Neuroscience, 11, 224-230.

Kristjánsson, Á., \& Nakayama, K. (2002). The attentional blink in time and space. Vision Research, 42, 2039-2050.

Marois, R., Chun, M. M., \& Gore, J. C. (2000). Neural correlates of the attentional blink. Neuron, 28, 299-308. 
Marois, R., \& Ivanoff, J. (2007). Capacity limits of information processing in the brain. Trends in Cognitive Science, 9, 296-305.

Marois, R., Yi, D. J., \& Chun, M. M. (2004). The neural fate of consciously perceived and missed events in the attentional blink. Neuron, 41, 465-472.

Masson, M. E. J., \& Loftus, G. R. (2003). Using confidence intervals for graphically based data interpretation. Canadian Journal of Experimental Psychology, 57, 203-220.

Nieuwenstein, M. R., Chun, M. M., van der Lubbe, R. H. J., \& Hooge, I. T. C. (2005). Delayed attentional engagement in the attentional blink. Journal of Experimental Psychology: Human Perception and Performance, 31, 1463-1475.

Olivers, C. N. L. (2004). Blink and shrink: The effect of the attentional blink on spatial processing. Journal of Experimental Psychology: Human Perception and Performance, 30, 613631.

Olivers, C. N. L., \& Meeter, M. (2008). A boost and bounce theory of temporal attention. Psychological Review, 115, 836-863.

Pashler, H. (1991). Shifting visual attention and selecting motor responses: Distinct attentional mechanisms. Journal of Experimental Psychology: Human Perception and Performance, 17, $1023-1040$.

Posner, M. I. (1980). Orienting of attention. The Quarterly Journal of Experimental Psychology, 32, 3-25.

Raymond, J. E., Shapiro, K. L., \& Arnell, K. M. (1992). Temporary suppression of visual processing in an RSVP task: An attentional blink? Journal of Experimental Psychology: Human Perception and Performance, 18, 849-860.

Robitaille, N., Jolicoeur, P., Dell'Acqua, R., \& Sessa, P. (2007). Shortterm consolidation of visual patterns interferes with visuo-spatial attention: Converging evidence from human electrophysiology. Brain Research, 1185, 158-169.

Santangelo, V., Olivetti Belardinelli, M., \& Spence, C. (2007). The suppression of reflexive visual and auditory orienting when attention is otherwise engaged. Journal of Experimental Psychology: Human Perception and Performance, 33, 137-148.

Santangelo, V., \& Spence, C. (2008). Is the exogenous orienting of spatial attention truly automatic? Evidence from unimodal and multisensory studies. Consciousness and Cognition, 17, 989-1015.

Shapiro, K. L., Hillstrom, A. P., \& Husain, M. (2002). Control of visuotemporal attention by inferior parietal and superior temporal cortex. Current Biology, 12, 1320-1325.

Theeuwes, J. (1991). Exogenous and endogenous control of attention: The effect of visual onsets and offsets. Perception \& Psychophysics, 49, 83-90.

Van Vleet, T., \& Robertson, L. (2006). Cross-modal interactions in time and apace: Auditory influence on visual attention in hemispatial neglect. Journal of Cognitive Neuroscience, 18, 1368-1379.

Visser, T. A. W., Zuvic, S. M., Bischof, W. F., \& Di Lollo, V. (1999). The attentional blink with targets in different spatial locations. Psychonomic Bulletin \& Review, 6, 432-436.

Yantis, S., \& Jonides, J. (1990). Abrupt visual onsets and selective attention: Voluntary versus automatic allocation. Journal of Experimental Psychology: Human Perception and Performance, $16,121-134$.

Zhang, D., Shao, L., Nieuwenstein, M., \& Zhou, X. (2008). Top-down control is not lost in the attentional blink: Evidence from intact endogenous cueing. Experimental Brain Research, 185, 287295. 